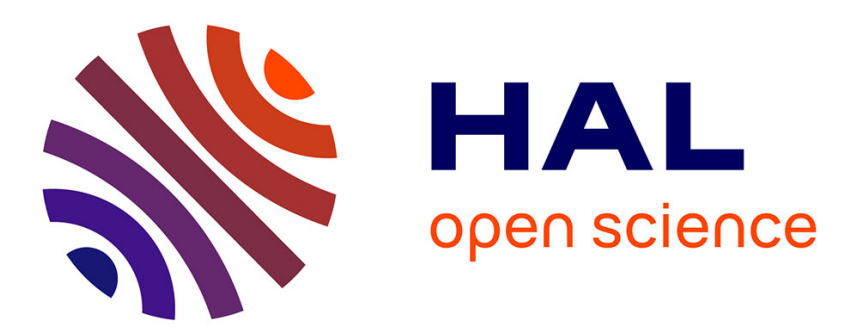

\title{
Timed synthesis control approach for tolerant-fault control of Discrete Event Systems (DES)
}

Imane Tahiri, Philippot Alexandre, V. Carre-Menetrier, A Tajer

\section{To cite this version:}

Imane Tahiri, Philippot Alexandre, V. Carre-Menetrier, A Tajer. Timed synthesis control approach for tolerant-fault control of Discrete Event Systems (DES). International Conference on Control, Automation and Diagnosis (ICCAD), 2018, Marrakech, Morocco. hal-02113921

\section{HAL Id: hal-02113921 \\ https://hal.science/hal-02113921}

Submitted on 29 Apr 2019

HAL is a multi-disciplinary open access archive for the deposit and dissemination of scientific research documents, whether they are published or not. The documents may come from teaching and research institutions in France or abroad, or from public or private research centers.
L'archive ouverte pluridisciplinaire HAL, est destinée au dépôt et à la diffusion de documents scientifiques de niveau recherche, publiés ou non, émanant des établissements d'enseignement et de recherche français ou étrangers, des laboratoires publics ou privés. 


\section{Timed synthesis control approach for tolerant-fault control of Discrete Event Systems (DES)}

\author{
I. Tahiri ${ }^{1 \& 2}$, A. Philippot ${ }^{1}$, V. Carré-Ménétrier ${ }^{1}$ \\ ${ }^{1}$ Research Centre in Information and Communication \\ Science and Technology (CReSTIC) \\ University of Reims Champagne Ardenne (URCA), \\ FRANCE \\ imane.tahiri@univ-reims.fr, alexandre.philippot@univ- \\ $\underline{\text { reims.fr, Veronique.carre@univ-reims.fr }}$
}

\author{
A. Tajer ${ }^{2}$ \\ ${ }^{2}$ LGECoS Laboratory \\ University Cadi Ayyad - ENSA Marrakech, MOROCCO \\ a.tajer@uca.ma
}

\begin{abstract}
This paper presents an approach of a safe control synthesis of Timed Discrete Event Systems, based on timed properties. This synthesis aims to realize a fault-tolerant control during the detection of sensor faults. To establish this synthesis, the proposed approach relies on the modularity of the manufacturing systems to avoid the combinatorial explosion recurrent to several approaches. An example of a manufacturing system illustrates our remarks.
\end{abstract}

Keywords - Timed discrete event systems, supervision theory, fault tolerant control, distributed control, manufacturing systems, modeling.

\section{Introduction}

A Discrete Event System (DES) is defined as a dynamic system that can be described in a discrete state space and whose evolution is described by state transitions triggered by events [1]. One of the recurring problems with these systems is the establishment of an adequate control law that guarantees the safety of equipment and personnel while performing the tasks required. In [2], Ramadge and Wonham $(\mathrm{R} \& \mathrm{~W})$ initiated the Supervisory Control Theory (SCT) of DES (fig.1).

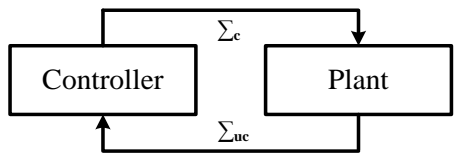

Fig. 1. Supervisory Control Theory principle

This approach consists of determining a controller (called supervisor) which has the effect of restricting all possible states of the system to a subset of permissible states. R\&W use finite state machines (FSM) and languages to model these systems. Although this approach is very formal, it shows a major disadvantage of its use on complex systems: the combinatorial explosion. In [3], [4], [5], [6] and [7], some approaches for control synthesis of DES have been proposed to overcome this problem. Although these approaches may have reduced the combinatorial explosion problem, they nevertheless remain dependent on Boolean information. But it is not the only information present when we consider time. This is called Timed Discrete Event Systems (TDES).
Several approaches of literature [8], [9], [10] and [11] use timed automata, Petri nets, or finite state machines as a TDES modeling tools. In these works, time is modeled in two ways:

- The discrete time, for which the progression of time is discrete and measured by a digital clock which is incremented by one unit at a time.

- The dense time, for which the progression of time is continuous and measured by clocks taking positive real values.

However, taking into account the passage of time makes the modeling step as well as the synthesis of the control by supervision more complex. To overcome these difficulties, we propose an extension of control synthesis approach proposed in [5] by exploiting its distributed design and introducing the time factor in the first stages of modeling.

The purpose of this proposal is to construct a control structure that can be used as a fault-tolerant control. Indeed, many industrial applications require fault tolerance and continuity of service. Therefore, some strategies are defined from the earliest stages of the control design to facilitate the detection of faults, their location and the control reconfiguration. Several approaches have been proposed to overcome this problem. In [12], the authors have defined a switch control formalism that revises the controller parameters when a change of an embedded sensor network occur or when it's repaired. The DES and its specification controller are modeled by a finite state machine. A mega-controller changes in the observation chains and selects the appropriate logic controller when changes occur. In [13] a reconfiguration approach based on supervisory control theory (SCT) was developed, the authors proposed a procedure based on SCT to reconfigure the controller without reconstructing its complete design. The reconfiguration keeps the behavior that does not change, eliminates the one that becomes useless after a faulty evolution, and adds the new updated behavior. Subsequently, the SCT ensures that the specifications are respected if a control synthesis is applied. Therefore, a partial reconfiguration using the SCT continues to adhere to the specifications that have not changed and ensures that the updated ones are also complied with in the new control law. In [14] a generic plant model of a 
DES that generates two behaviors is formulated: a nominal one and a failing one under a set of functional specifications and security constraints. The approach proposed an active faulttolerant control architecture such that the supervisor actively responds to faults as follows: (i) Design a nominal supervisor imposing faultless specifications, (ii) Design a diagnoser that unambiguously detects faults, and finally (iii) plant automatic switching to the second supervisor after a fault detection such that the system continues to operate safely.

However, the use of these different methods is not easy. Indeed, the construction of fault-tolerant supervisors is a difficult task when the models to be treated are presented under a large size. This disadvantage is generally due to the fact that most of the approaches proposed in the literature for faulttolerant control have been developed for systems where the information used is decentralized, centralized or hierarchical. However, the majority of manufacturing systems, are informally distributed.

Therefore, our contribution will be a fault-tolerant control method (fig.2). based on this axis of distributivity. When a sensor fault appears thanks to the diagnostic module, the information lost on a sensor will be replaced by a timed information through a coordinator that allows the switch between the distributed controller $\left(\mathrm{G}_{\mathrm{DCi}}\right)$ interpreted by a grafcet modelling (IEC 60848) of the normal behavior mode and the reconfigured one $\left(\mathrm{G}_{\mathrm{TDCi}}\right)$ in order to guarantee the continuation of the plant tasks in spite of the faults which may hinder its normal functioning. The diagnoser and coordinator determination is not the aim of this work, so we opt for the approach evoked in [15] to diagnose our system, and a method for determining the coordinator will be proposed in future works.

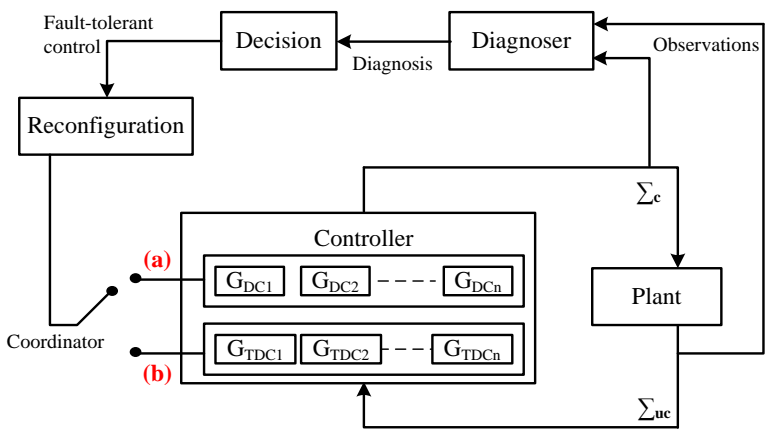

Fig. 2. A fault-tolerant control loop $3)$ :

The proposed approach consists of the following steps (Fig.

1. Modular and timed plant modeling: the resulting automaton is a timed plant element $\mathrm{G}_{(\mathrm{TPE})}$.

2. Definition of local functional and safety constraints in order to synthesis a local controller: the resulting automaton is a timed local controller $\mathrm{G}_{(\mathrm{TLC})}$.

3. Taking into account overall behavior constraints to determine timed distributed controllers: the resulting automaton is a $\mathrm{G}_{(\mathrm{TDC})}$.
4. Interpretation of timed distributed controllers into grafcet specification and their implementation in a programmable logic controller (PLC) through a standard language (IEC 61131-3).

The paper is organized as following: Each step of the proposed approach is described in Section 2. Section 3 illustrates our results around a transfer system benchmark. Conclusions and discussion close the paper in section 4 .

\section{Basic Concept of Distributed Approach of TDESs}

\section{A. Synthesis of timed local supervisory control}

Based on works evoked in [5] and [16], we propose an extended approach to synthesis timed local controllers TLC, with a method allowing their obtaining using Supremica software by the following steps:

- Define the timed plant element (TPE) automaton $\mathrm{G}_{(\mathrm{TPE})}$.

- Define an extended model of $\mathrm{G}_{(\mathrm{TPE})}$.

- Define the set of local specifications.

- Synthesize the timed local controllers $\mathrm{G}_{(\mathrm{TLC})}$.

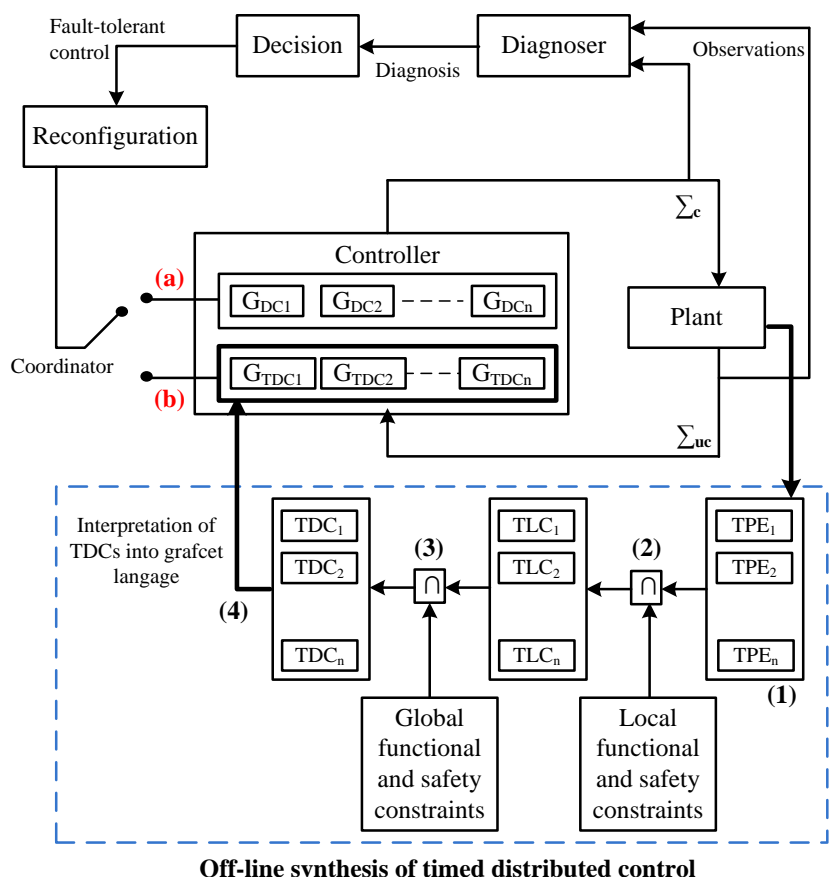

Fig. 3. The proposed distributed supervisory control architecture

The timed plant modeling is an extension of the practical model [17] that describe the most respectful behavior of a component by introducing time.

A Plant Element (PE) is obtained by a synchronous composition of detector, pre-actuators and actuators models. Each PE does not always have a set of detectors sufficient to be described. Therefore, a timed detectors models are introduced. It is then necessary to determine a timed actuators models.

Formally, if the timed detectors model is modeled by an automaton $\mathrm{G}_{(\mathrm{TD})}$ and the timed actuators model is defined by an 
automaton $\mathrm{G}_{(\mathrm{TA})}$, The TPE automaton' model is obtained by a synchronous composition of these two models:

$\mathrm{G}_{(\mathrm{TPE})}=\left(\mathrm{S}, \Sigma, \mathrm{T}, \mathrm{D}, \delta, \mathrm{s}_{0}, \mathrm{~S}_{\mathrm{m}}\right)=\mathrm{G}_{(\mathrm{TD})} \| \mathrm{G}_{(\mathrm{TA})}$

With:

- $\mathrm{S}=$ Finite set of states

- $\Sigma=$ Finite set of events such as: $\Sigma=\Sigma_{\mathrm{c}} \cup \Sigma_{\mathrm{uc}}$. With:

$\Sigma_{\mathrm{c}}=\uparrow \downarrow \mathrm{Z} \cup \uparrow \downarrow \mathrm{T} \cup \mathrm{D}$ and $\Sigma_{\mathrm{uc}}=\uparrow \downarrow \mathrm{E}$

$\uparrow \downarrow Z$ : Set of activations and deactivations of control orders $\mathrm{Z}_{\mathrm{i}}$ (actuators outputs).

$\uparrow \downarrow E$ : Set of rising and falling edges of inputs variables $e_{i}$ (sensors inputs).

$\uparrow \downarrow T$ : Set of activations and deactivations of clocks $T_{i}$.

With: $\Sigma_{\mathrm{nT}}=\uparrow \downarrow \mathrm{Z} \cup \uparrow \downarrow \mathrm{E}$ set of not-timed events, and $\Sigma_{\mathrm{T}}$ $=\uparrow \downarrow T \cup D$ set of timed events.

- $\mathrm{T}$ : Set of clocks, such as $\mathrm{T}=\uparrow \mathrm{ti} \cup \downarrow_{\mathrm{ti}}$.

- D: Finite set of durations di associated to each clock ti, such as $D=\left\{d_{1}, d_{2}, \ldots, d_{i}\right\}$.

- $\delta$ : Transition function. A transition is defined by: $\delta\left(\mathrm{s}_{1}, \sigma\right)=\mathrm{s}_{2}$ such that $s_{1}$ is the initial state and $s_{2}$ is the final state. $\sigma$ is the occurrence of a timed event or not of $\Sigma$.

- $\mathrm{s}_{0}$ : Initial state

- $\mathrm{S}_{\mathrm{m}}$ : Set of marked states

Once $G_{(T P E)}$ is obtained, its extended automata is determined through an Extended Finite State Machines EFSM generated by supremica [16]. Differently to finite state machines (FSM), EFSM uses variables, guards and actions that can help the operator to compactly represent a large and complicated DES. Guard expressions are used to restrict the behavior of the system and action functions are used to update variables. The resulting extended automata of $\mathrm{G}_{(\mathrm{TPE})}$ generated by supremica is noted $\mathrm{G}_{(\mathrm{TPE})}$ ccurr.

A TLC is obtained using models of the TPE and its associated set of specifications, such that it is minimally restrictive with respect to the plant behavior, while still guaranteeing that the specifications are upheld.

These specifications are defined by a logical implication in the form of:

$$
x \Rightarrow \bar{y}
$$

With $x \in S_{i}$ and $y \in \Sigma_{c}$.

The implication (1) means that if $x$ is true then $y$ is forbidden, which is proved that can be also interpreted in the following form [18]:

$$
x \cdot y=0
$$

In this paper we choose equation (2) to interpret the set of specifications related to each TPE, and for an implementation purpose, the set of specifications is defined as an (EFSM) as shown in fig.4.

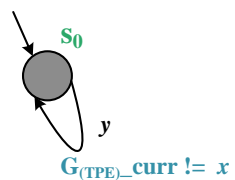

Fig. 4. EFSM specification example

The EFSM specification is composed of a single state and a self-loop transition. A guard " $\mathrm{G}_{(\mathrm{TPE})}$ curr ! = $\mathrm{x}$ " ( the courant state is different to $\mathrm{x}$ ) is associated to the transition labeled with event $y$, the transition is enabled if and only if the guard formula is true. And by applying the synthesis of supervisory control on supremica of the two automata presenting $G_{(T P E)}$ and specifications. we obtain a $\mathrm{G}_{(\mathrm{TLC})}$.

\section{B. Synthesis of timed global supervisory control}

To pass from a TLC to a timed distributed controller (TDC), it is necessary to go through two steps: the first one is the aggregation of the controllable events in macro-states and the second one is the integration of the functional and security constraints of the overall behavior. An extract of a TLC automaton is shown in fig. 5 .

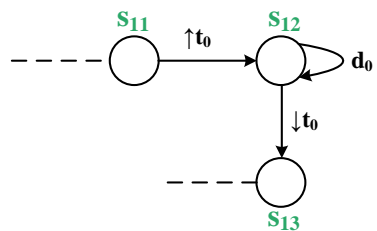

Fig. 5. Extract from a timed local controller automaton $\mathrm{G}_{(\mathrm{TLC})}$

The aggregation of a TLC is carried out in two steps:

Step 1: Associate the states reached by the controllable events $\left(\uparrow \downarrow_{\mathrm{Z}_{\mathrm{i}}} \in\left\{\Sigma_{\mathrm{c}} \cap \Sigma_{\mathrm{nT}}\right\}\right)$ in macro-states linked by uncontrollable events $\left(\mathrm{e}_{\mathrm{i}} \in \Sigma_{\mathrm{uc}}\right)$, and timed events: $\left(\uparrow \downarrow_{\mathrm{t}_{\mathrm{i}}} \in \Sigma_{\mathrm{T}}\right) \cup\left(\mathrm{d}_{\mathrm{i}} \in \Sigma_{\mathrm{T}}\right)$. If the TLC' state is associated to a rising edge of a controllable event, then the order is authorized and belongs to the set Ord. If it's associated to a falling edge of this event then the order is inhibited and belongs to the set Inh.

Step2: Aggregate timed events $\uparrow \downarrow_{t_{i}}$ in macro-states linked by uncontrollable events $\left(\mathrm{e}_{\mathrm{i}} \in \Sigma_{\mathrm{uc}}\right)$ and timed events $\mathrm{d}_{\mathrm{i}}$. If the state of the timed local aggregated controller by the first aggregation is reached by an event corresponding to the clock' activation, then this event belongs to a set noted $\mathrm{A}_{\text {clock. }}$. If it is reached by an event corresponding to the clock' deactivation, then this event belongs to a set noted $\mathrm{D}_{\text {clock}}$. The self-loop transition will be the transition that links the two macro-states that contain the two sets $\left(\mathrm{A}_{\text {clock }}\right.$ and $\left.\mathrm{D}_{\text {clock }}\right)$. The resulting automaton is the timed local aggregated controller called TLAC. An extract of TLAC automaton is shown in fig. 6 .

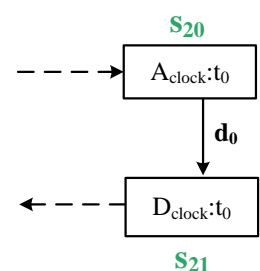

Fig. 6 Extract from a timed local aggregated controller automaton $\mathrm{G}_{(\mathrm{TLAC})}$ 
The TLCs behave locally while respecting local security constraints. In order for these controllers to communicate and synchronize with each other, a set of global functional and security constraints must be determined in order to obtain timed distributed controllers TDC able of behaving in both local and global contexts, while ensuring the security of each plant element. A global constraint is modelled as follows:

$$
\text { If a then }\left\{\begin{array}{l}
b=1 \\
b=0
\end{array}\right.
$$

If " $b$ " is allowed and belongs to the set Ord, then $(b=1)$ if and only if the condition " $a$ " is true.

If " $b$ " is inhibited and belongs to the set Inh, then $(b=0)$ if and only if the condition " $a$ " is true.

A TDC automaton is formally defined by: $G_{(T D C)}=\left(S_{(T D C)}\right.$, $\left.\Sigma_{(\mathrm{TDC})}, \mathrm{D}_{(\mathrm{TDC})}, \delta_{(\mathrm{TDC})}, \mathrm{E}_{(\mathrm{TDC})}, \mathrm{C}_{(\mathrm{TDC})}, \mathrm{S}_{0(\mathrm{TDC})}, \mathrm{S}_{\mathrm{m}(\mathrm{TDC})}\right)$, with:

- $\mathrm{S}_{(\mathrm{TDC})}$ : Finite set of states.

- $\Sigma_{(\mathrm{TDC})}$ : Finite set of events such as $\Sigma_{(\mathrm{TDC})}=\left\{\Sigma_{\mathrm{uc}(\mathrm{TDC})} U\right.$ $\left.\Sigma_{\mathrm{c}(\mathrm{TDC})}\right\}=\left\{\Sigma_{\mathrm{T}(\mathrm{TDC})} \cup \Sigma_{\mathrm{nT}(\mathrm{TDC})}\right\} \neq \emptyset$.

- $\mathrm{D}_{\text {(TDC) }}$ : Finite set of durations $\mathrm{d}_{\mathrm{i}}$ associated to each clock $\mathrm{t}_{\mathrm{i}}$.

- $\delta_{\text {(TDC) }}$ : Transition function. A transition is defined by: $\delta\left(s_{1}, \sigma\right)=s_{2}$ such that $s_{1}$ is the initial state and $s_{2}$ is the target state. $\sigma$ is the occurrence of a timed event $d_{i}$ or uncontrollable event $\mathrm{e}_{\mathrm{i}}$.

- $\mathrm{E}_{(\mathrm{TDC})}$ : Set associated to each state of $\mathrm{S}_{(\mathrm{TDC})}, E_{T D C}^{s_{i}}=\left\{O r d^{s_{i}}\right.$, $\left.\operatorname{Inh}^{s_{i}}, A_{\text {Clock }}^{s_{i}}, D_{\text {Clock }}^{s_{i}}\right\}$.

- $\mathrm{C}_{(\mathrm{TDC})}$ : Finite set of constraints allowing authorization or inhibition of an order $\mathrm{Z}_{\mathrm{i}}$.

The example of Fig.7 illustrates a TDC automaton. Each rectangle represents a state with its associated actions (authorised and inhibited orders), clocks (activated and deactivated clocks), and conditions, if any.

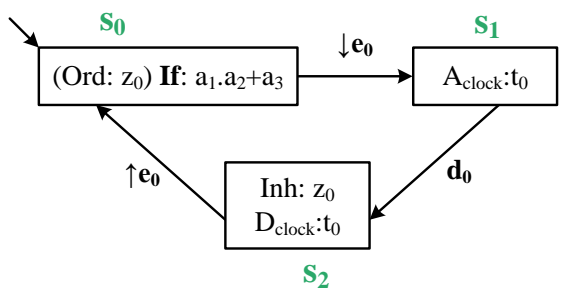

Fig. 7. Example of a timed distributed controller automaton $G_{(T D C)}$

The set of $\mathrm{S}_{\text {(TDC) }}$ states is defined by $\left\{\mathrm{s}_{0}, \mathrm{~s}_{1}, \mathrm{~s}_{2}\right\}$, the initial state $\mathrm{S}_{0(\mathrm{TDC})}$ is identified by an incoming arrow. $\Sigma_{(\mathrm{TDC})}=\left\{\Sigma_{\mathrm{T}(\mathrm{TDC})} \cup \Sigma_{\mathrm{nT}(\mathrm{TDC})}\right\}$ with $\Sigma_{\mathrm{nT}(\mathrm{TDC})}=\left\{\uparrow \downarrow_{\mathrm{e}_{0}}, \uparrow \downarrow_{\mathrm{z}_{0}}\right\}$ is the set of non-timed events and $\Sigma_{\mathrm{T}(\mathrm{TDC})}=\left\{\uparrow \downarrow_{\mathrm{t}_{0}}, \mathrm{~d}_{0}\right\}$ is the set of timed events. $E_{T D C}^{s_{0}}=\left\{\mathrm{z}_{0}, \emptyset, \emptyset, \emptyset\right\}, E_{T D C}^{s_{1}}=\left\{\varnothing, \emptyset, \mathrm{t}_{0}, \emptyset\right\}, E_{T D C}^{2}=$ $\left\{\varnothing, \mathrm{z}_{0}, \emptyset, \mathrm{t}_{0},\right\}$. Empty actions, clocks and conditions are not represented.

\section{Grafcet interpretation of the TDC}

For an implementing purpose of timed distributed controllers in a PLC, an approach is followed to translate them into grafcet language.

The clock activation $\left(A_{\text {clock}}: t_{i}\right)$ is translated by a timed action $t_{i}$, associated to the grafcet step $X_{i}$, and the duration $d_{i}$ is transformed into transition receptivity after $X_{i}$. The clock deactivation is not introduced in the grafcet because once the duration elapses, it causes the passage to the next step $X_{i+1}$. An example of this interpretation is shown in Fig. 8

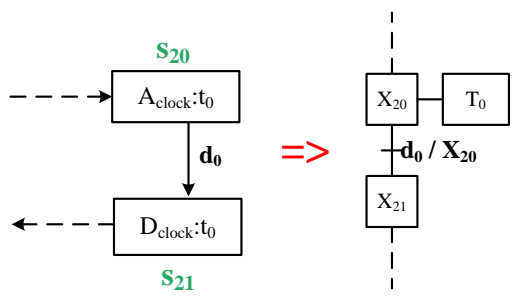

Fig. 8. example of timed distributed controller interpretation

\section{Application to a manufacturing system}

To illustrate our contribution, we use 3D FACTORY I/O simulator (https://factoryio.com/). It's a digital twin for learning automation technologies, designed to be easy to use, it allows to quickly build a virtual factory using a selection of common industrial parts. FACTORY I/O also includes many scenes inspired by typical industrial applications, ranging from beginner to advanced difficulty levels. Using this tool, we built a boxes transfer system (fig.9), which is composed of:.



Fig. 9. The manufacturing systems

- Two pushers (monostable single effect cylinders): The first one $\left(\mathrm{P}_{1}\right)$ pushes boxes in front of the second one $\left(\mathrm{P}_{2}\right)$ which transfers them to the evacuation conveyor.

- Two conveyor belts, to transport boxes in front the pusher $\mathrm{P}_{1}$, and to evacuate them to the stock.

- Six sensors: $\left\{\mathrm{e}_{0}, \mathrm{e}_{1}\right\}$ sensors for the presence of a box in front of the pushers, $\left\{\mathrm{e}_{2}, \mathrm{e}_{4}\right\}$ sensors associated to the pushers input, and $\left\{\mathrm{e}_{3}, \mathrm{e}_{5}\right\}$ sensors associated to the pushers output.

- A start' push button $\mathrm{Pb}_{\text {Start. }}$

In this example we assume that the sensor $\mathrm{e}_{3}$ don't work anymore and must be replaced by a timed information. the element that allows us to ensure this information is the clock $t_{1}$. In the following figure (fig.10) we present the two models of the system with and without sensor $e_{3}$. We then show that the information lost on $\mathrm{e}_{3}$ due to a fault is replaced by a clock. the flow of the predetermined duration means that the pusher has reached its final output position. 
In order to obtain a timed local controller that describes the desired local behavior, some functional specifications must be determined:

- First specification: The deactivation of $\mathrm{P}$ is due to the clock deactivation, so $\downarrow P$ must be forbidden to appear in state $s_{1}$ and $s_{3}$. This specification is defined by the following equation (3).
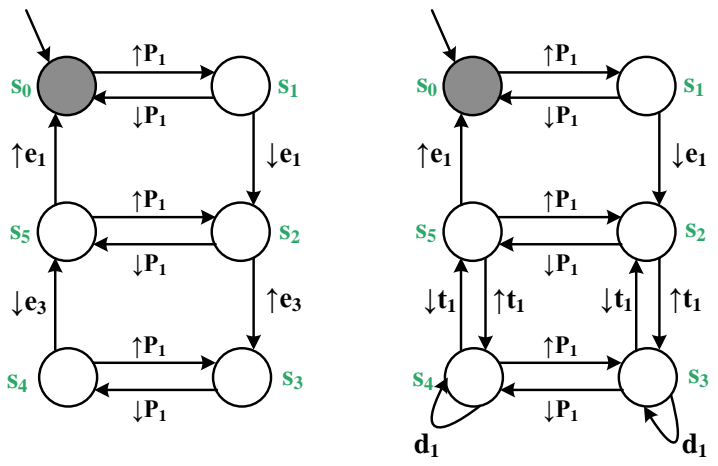

Fig. 10. Timed plant element automaton corresponding to the pusher $1 \mathrm{G}_{(\mathrm{TPE}) \mathrm{P} 1}$

$$
\left(s_{1}+s_{3}\right) \cdot \downarrow P_{1}=0 \Leftrightarrow\left\{\begin{array}{l}
s_{1} \cdot \downarrow P_{1}=0 \\
s_{3} \cdot \downarrow P_{1}=0
\end{array}\right.
$$

The automaton that translates this specification is shown in fig. 11 .



Fig. 11. Specification 1 automaton

The figure above shows that if the current state of $\mathrm{G}_{\text {(TPE)P1 }}$ is different from $s_{1}$ and $s_{3}$, then $\downarrow P_{1}$ is allowed to appear.

- Second specification: The deactivation of $t_{1}$ is due to the sensor $\mathrm{e}_{1}$ deactivation, so $\downarrow_{\mathrm{t}_{1}}$ must be forbidden to appear in state $s_{5}$. This specification is defined by the following equation.

$$
s_{5} \cdot \downarrow t_{1}=0
$$

The automaton that translates this specification is shown in fig. 12 .

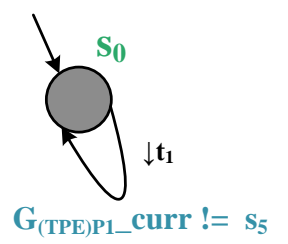

Fig. 12. Specification 2 automaton
Applying these two specifications, the two transitions associated to $\downarrow \mathrm{P}_{1}$ and outgoing from $\mathrm{s}_{1}$ and $\mathrm{s}_{3}$, and the transition associated to $\downarrow t_{1}$ and outgoing from $s_{5}$ are deleted. Consequently, the state $\mathrm{s}_{4}$ becomes inaccessible and will be also deleted. The timed local controller describing the desired behavior obtained is shown in figure 13 .

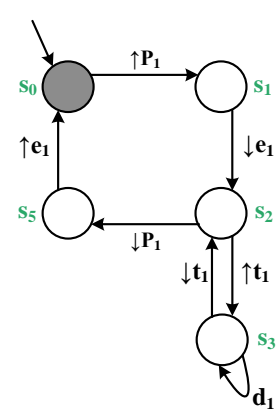

Fig. 13. The timed local controller automaton of pusher $1, \mathrm{G}_{(\mathrm{TLC}) \mathrm{P} 1}$

Applying the untimed and timed aggregation explained in previous sections, we obtain the following automaton:

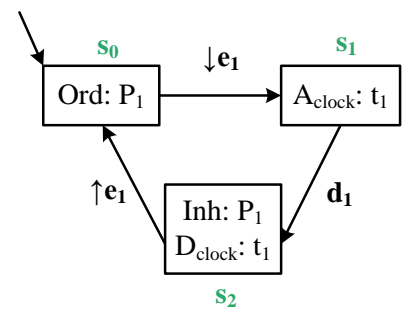

Fig. 14. Timed local aggregated controller of pusher $1 \mathrm{G}_{(\mathrm{TLAC}) \mathrm{P} 1}$

In the initial state $\mathrm{s}_{0}, \mathrm{P}_{1}$ is authorized and belongs to the set Ord of the authorized events, the deactivation of $\mathrm{e}_{1}$ causes the activation of the clock $t_{1}$, this activation is aggregated in a macro-state $s_{1}$ and belongs to the set $A_{\text {clock }}$ of clocks activation. After the flow of the duration $\mathrm{d}_{1}$ associated to the clock $\mathrm{t}_{1}, \mathrm{t}_{1}$ will be deactivated and will cause the deactivation of $\mathrm{P}_{1}$. Therefore, in the state $s_{2}, t_{1}$ will belong to the set $D_{\text {clock }}$ (set of clocks deactivation) and $\mathrm{P}_{1}$ will belong to the set Inh (Set of inhibited orders).

To be able to obtain the distributed controller corresponding to the pusher $\mathrm{P}_{1}$, the overall functional and security constraints must be taken into account. The pusher must push out if a piece is present $\left(\mathrm{e}_{0}=1\right)$, but to guarantee the safety, it must be verified that the pusher 2 is in the retracted position which is ensured by $\left(e_{4}=1\right)$. Therefore, these constraints will be translated by the following equation:

$$
\text { If }\left(\mathrm{e}_{0} \cdot \mathrm{e}_{4}\right) \text { then } \mathrm{P}_{1}=1
$$

Taking into account this specification, we obtain the following timed distributed controller automaton $\mathrm{G}_{(\mathrm{TDC}) \mathrm{P} 1}$. 


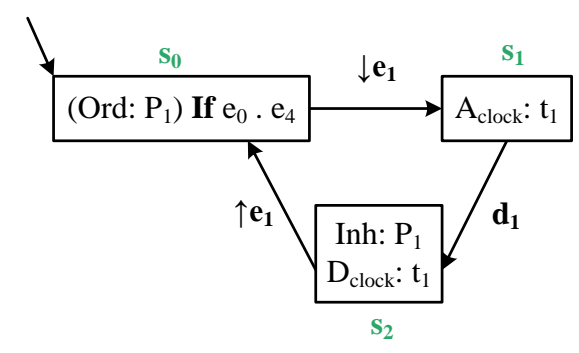

Fig. 15. Timed distributed controller of pusher 1

If the overall behavior specifications are verified, the output of pusher $P_{1}$ is allowed, which causes the deactivation of sensor $\mathrm{e}_{1}$ associated to $\mathrm{P}_{1}$ input. Once $\mathrm{e}_{1}$ is deactivated, the clock is activated until the passage of the duration $d_{1}$ which leads to $t_{1}$ and $P_{1}$ deactivation. Therefore, $P_{1}$ returns to its initial state and $\mathrm{e}_{1}$ will be reactivate.

The grafcet equivalent of this TDC automaton is shown in fig 16.

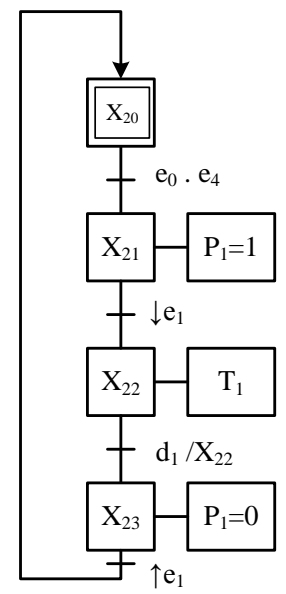

Fig. 16.Grafcet interpretation of $\mathrm{G}_{(\mathrm{TDC}) \mathrm{P} 1}$

\section{Conclusion and prospects}

In this work we have proposed a new approach of integrating time in order to synthesis a timed distributed control for discrete event systems DES. This control synthesis aims at achieving a fault-tolerant control during the detection of a sensor fault through the diagnoser. A decision block decides whether the fault is tolerable or not. If it is tolerable, a reconfiguration where the information lost on the sensor is replaced by a timed information is necessary. Our timed distributed control is based first on a timed modelling of each plant element, the second step is determining the different timed local controllers by taking account the local functional and security constraints. In order to define timed distributed controllers, the overall functional and security constraints should be verified. The resulting controllers are interpreted into grafcet language for an implementation purpose. In our shortterm works, a formal method to determine the coordinator that allows the switching between the normal behavior controller to its equivalent reconfigured behavior controller will be proposed.

\section{References}

[1] C. G. Cassandras., and S.Lafortune. « Introduction to Discrete Event Systems». 2nd ed. New York: Springer.10.1007/978-0-387-68612-7.

[2] P. J. G. Ramadge et W. M. Wonham, « The control of discrete event systems », Proc. IEEE, vol. 77, nº 1, p. 81-98, janv. 1989.

[3] M. Sayed-Mouchaweh, A. Philippot, et V. Carre-Menetrier, «Decentralized diagnosis based on Boolean discrete event models: application on manufacturing systems », Int. J. Prod. Res., vol. 46, n 19, p. 5469-5490, oct. 2008 .

[4] K. Cai et W. M. Wonham, «Supervisor Localization: A Top-Down Approach to Distributed Control of Discrete-Event Systems », IEEE Trans. Autom. Control, vol. 55, n 3, p. 605-618, mars 2010.

[5] Y. Qamsane, A. Tajer, et A. Philippot, «A synthesis approach to distributed supervisory control design for manufacturing systems with Grafcet implementation », Int. J. Prod. Res., sept. 2016.

[6] W. M. Wonham et P. J. Ramadge, « Modular supervisory control of discrete-event systems », Math. Control Signals Syst., vol. 1, n ${ }^{\circ} 1$, p. 13 30, févr. 1988.

[7] A. Khoumsi et H. Chakib, « Decentralized supervisory control of discrete event systems moving decisions closer to actions », in 2014 11th International Conference on Informatics in Control, Automation and Robotics (ICINCO), 2014, vol. 02, p. 280-287.

[8] J. S. Ostroff, « Deciding properties of timed transition models », IEEE Trans. Parallel Distrib. Syst., vol. 1, nº 2, p. 170-183, avr. 1990.

[9] B. A. Brandin et W. M. Wonham, «Supervisory control of timed discrete-event systems », IEEE Trans. Autom. Control, vol. 39, n 2, p. 329-342, févr. 1994.

[10] A. T. Sava et H. Alla, « A control synthesis approach for time discrete event systems », Math. Comput. Simul., vol. 70, nº 5, p. 250-265, févr. 2006.

[11] A. Khoumsi et L. Ouédraogo, « New results in modeling and supervisory control of real-time discrete event systems », in 2010 18th Mediterranean Conference on Control Automation (MED), 2010, p. 669-676.

[12] H. Darabi, M. A. Jafari, et A. L. Buczak, « A control switching theory for supervisory control of discrete event systems », IEEE Trans. Robot. Autom., vol. 19, nº 1, p. 131-137, févr. 2003.

[13] G. Faraut, L. Piétrac, et E. Niel, «Control law synthesis and reconfiguration using SCT », in 2010 Conference on Control and FaultTolerant Systems (SysTol), 2010, p. 576-581.

[14] J. Dai, A. Karimoddini, et H. Lin, « Achieving fault-tolerance and safety of discrete-event systems through learning », in 2016 American Control Conference (ACC), 2016, p. 4835-4840.

[15] A. Philippot et V. Carré-Ménétrier, «Methodology to obtain local discrete diagnosers: Submission for special session on diagnosis of DES: Application on a benchmark », in 2011 3rd International Workshop on Dependable Control of Discrete Systems, 2011, p. 47-52.

[16] K. Akesson, M. Fabian, H. Flordal, et R. Malik, «Supremica - An integrated environment for verification, synthesis and simulation of discrete event systems », in 2006 8th International Workshop on Discrete Event Systems, 2006, p. 384-385.

[17] A. Philippot. "Contribution Au Diagnostic Décentralisé Des Systèmes À Événements Discrets: Application Aux Systèmes Manufacturiers.” PhD thesis, Université de Reims-Champagne Ardenne, 2006.

[18] B. Riera, A. Philippot, R. Coupat, F. Gellot, et D. Annebicque, « A nonintrusive method to make safe existing PLC Program », IFAC-Pap., vol. $48, \mathrm{n}^{\circ} 21$, p. 320-325, janv. 2015. 